\title{
Urban and rural differences in geographical accessibility to inpatient palliative and end-of-life (PEoLC) facilities and place of death: a national population-based study in England, UK
}

Emeka Chukwusa ${ }^{1 *} \mathbb{D}$, Julia Verne ${ }^{2}$, Giovanna Polato ${ }^{3}$, Ros Taylor ${ }^{4,5}$, Irene J Higginson ${ }^{1}$ and Wei Gao ${ }^{1}$

\begin{abstract}
Background: Little is known about the role of geographic access to inpatient palliative and end of life care (PEoLC) facilities in place of death and how geographic access varies by settlement (urban and rural). This study aims to fill this evidence gap.

Methods: Individual-level death data in 2014 ( $N=430,467$, aged 25+) were extracted from the Office for National Statistics (ONS) death registry and linked to the ONS postcode directory file to derive settlement of the deceased. Drive times from patients' place of residence to nearest inpatient PEoLC facilities were used as a proxy estimate of geographic access. A modified Poisson regression was used to examine the association between geographic access to PEoLC facilities and place of death, adjusting for patients' socio-demographic and clinical characteristics. Two models were developed to evaluate the association between geographic access to inpatient PEoLC facilities and place of death. Model 1 compared access to hospice, for hospice deaths versus home deaths, and Model 2 compared access to hospitals, for hospital deaths versus home deaths. The magnitude of association was measured using adjusted prevalence ratios (APRs).

Results: We found an inverse association between drive time to hospice and hospice deaths (Model 1), with a doseresponse relationship. Patients who lived more than 10 min away from inpatient PEoLC facilities in rural areas (Model 1: APR range 0.49-0.80; Model 2: APR range 0.79-0.98) and urban areas (Model 1: APR range 0.50-0.83; Model 2: APR range $0.98-0.99$ ) were less likely to die there, compared to those who lived closer (i.e. $\leq 10$ min drive time). The effects were larger in rural areas compared to urban areas.

Conclusion: Geographic access to inpatient PEoLC facilities is associated with where people die, with a stronger association seen for patients who lived in rural areas. The findings highlight the need for the formulation of end of life care policies/strategies that consider differences in settlements types. Findings should feed into local end of life policies and strategies of both developed and developing countries to improve equity in health care delivery for those approaching the end of life.
\end{abstract}

Keywords: Place of death, Rural-urban, Geographic accessibility, Inpatient palliative and end of life care (PEoLC) facilities

\footnotetext{
*Correspondence: emeka.chukwusa@kcl.ac.uk

${ }^{1}$ Department of Palliative Care, Policy and Rehabilitation, Cicely

Saunders Institute, King's College London, Bessemer Road, Denmark Hill,

London SE5 9PJ, UK

Full list of author information is available at the end of the article
} 


\section{Background}

Place of death has been an important proxy outcome measure of end of life care [1,2] and can potentially determine the quality of care patients receive prior to death. Access to palliative care is important [3, 4] and a priority of the World Health Organisation, who made a global resolution in 2014, calling on member states to improve access to palliative care as a core component of health [5]. In order to achieve this goal, an understanding of the role of geographic access to inpatient PEoLC facilities in place of death is essential for service improvement, development and planning.

A growing body of international and UK studies have found considerable rural-urban variations in place of death. In general, researchers have found that there is limited use of PEoLC services in rural areas prior to death [6-8] and rural dwellers are more likely to die in hospices or at home compared to urban dwellers [9-13]. These variations suggest that rural and urban dwellers experience different patterns of geographic access to PEoLC facilities.

Access is a complex and multidimensional concept [14-16] comprising of five dimensions; availability, accessibility, affordability, acceptability, and accommodation $[14,17]$. Access can also be classified into potential and realised access. Realised access is the actual utilisation of health services, whereas potential access quantifies the propensity to utilise health services [18]. Geographic access hugely influences health service utilisation and outcomes [19-22], but, little is known about the role of geographic access to PEoLC in place of death.

Previous PEoLC accessibility studies in the UK and elsewhere have focused largely on mapping equity of PEoLC provision $[23,24]$ quantifying proximity to PEoLC [25], identifying underserved or shortage areas of PEoLC facilities [26, 27], characterising of variations in access $[23,28]$ and location planning or site suitability assessment [29-31]. None of these studies have examined the role of geographic access to PEoLC in place of death. Further, most PEoLC accessibility studies [23, 24, 28, 31, 32] used aggregate-level data as the unit of analysing geographic access by measuring distance or travel time from centroids of census areas, blocks or dissemination area to the nearest PEoLC facilities. The use of aggregate-level data for accessibility analysis can be prone to errors [33, 34 ] and can potentially lead to inappropriate policies. The study by Gatrell and colleagues [35], is an exception. The authors examined the role of geographic access to PEoLC facilities in place of death, using cancer only data from a specific health authority in North West England, UK and they did not control for important factors (e.g. number of contributory causes of death and region of residence) known to influence place of death in previous studies.
Further, the study did not consider whether access varies between rural and urban areas, thus limiting the generalisability of their findings.

Our study addressed these research gaps by using large individual-level death data (including deaths from cancers and non-cancers) to explore the urban-rural differences in the association between geographic access to inpatient PEoLC facilities in England, UK. Understanding the role of geographic access in place of death is the first step to providing quality palliative care. Knowledge of the role of geographic access and how geographic access differs between urban and rural areas can provide an evidence-base for developing robust service improvement interventions to improve access to PEoLC.

\section{Methods \\ Study design and setting}

A national population-based observational study in England, UK.

\section{Data sources and study population}

Death data consisting of all deaths from non-accidental causes of patients aged 25 years and above in England, 2014, were extracted from the Office for National Statistics (ONS) death registry. The ONS death registration data included the age and gender of the deceased, the postcode of place of death (i.e. hospital, own home, care home, hospice etc.), the postcode of usual place of residence, the underlying Cause of Death (CoD), and the Number of Contributory causes of Death (NCoD). Death data were linked to the ONS postcode directory file [36] to derive the settlement of the deceased. Settlement classification in the ONS postcode directory file was based on the 2011 Rural-Urban Classification (RUC2011) [37]. The RUC2011 defines an urban area as a settlement with a population of 10,000 people or more [37]. The linkage of death data was based on patients' postcode of residence.

Hospital location data were downloaded from NHS digital (https://digital.nhs.uk/) [38], and the National End of Life Care Intelligence Network website (http://www. endoflifecare-intelligence.org.uk/home) [39]. Hospice location data were provided by Hospice UK and supplemented with data from the UK Hospice aid directory website (http://www.hospiceaid.org.uk/images/guide _to_hospice_aid/hospicedirectorybycounty.pdf) [40]. Hospice data used in this study comprised of hospices providing adult inpatient services. Children's hospices were excluded from the datasets. Figure 1 shows the spatial distribution of hospices and hospitals in England, UK. Road data used for measuring geographic access was the Ordnance MasterMap ${ }^{\circledR}$ integrated transport network 
- Hospices

- Hospitals

Rural areas

Urban areas

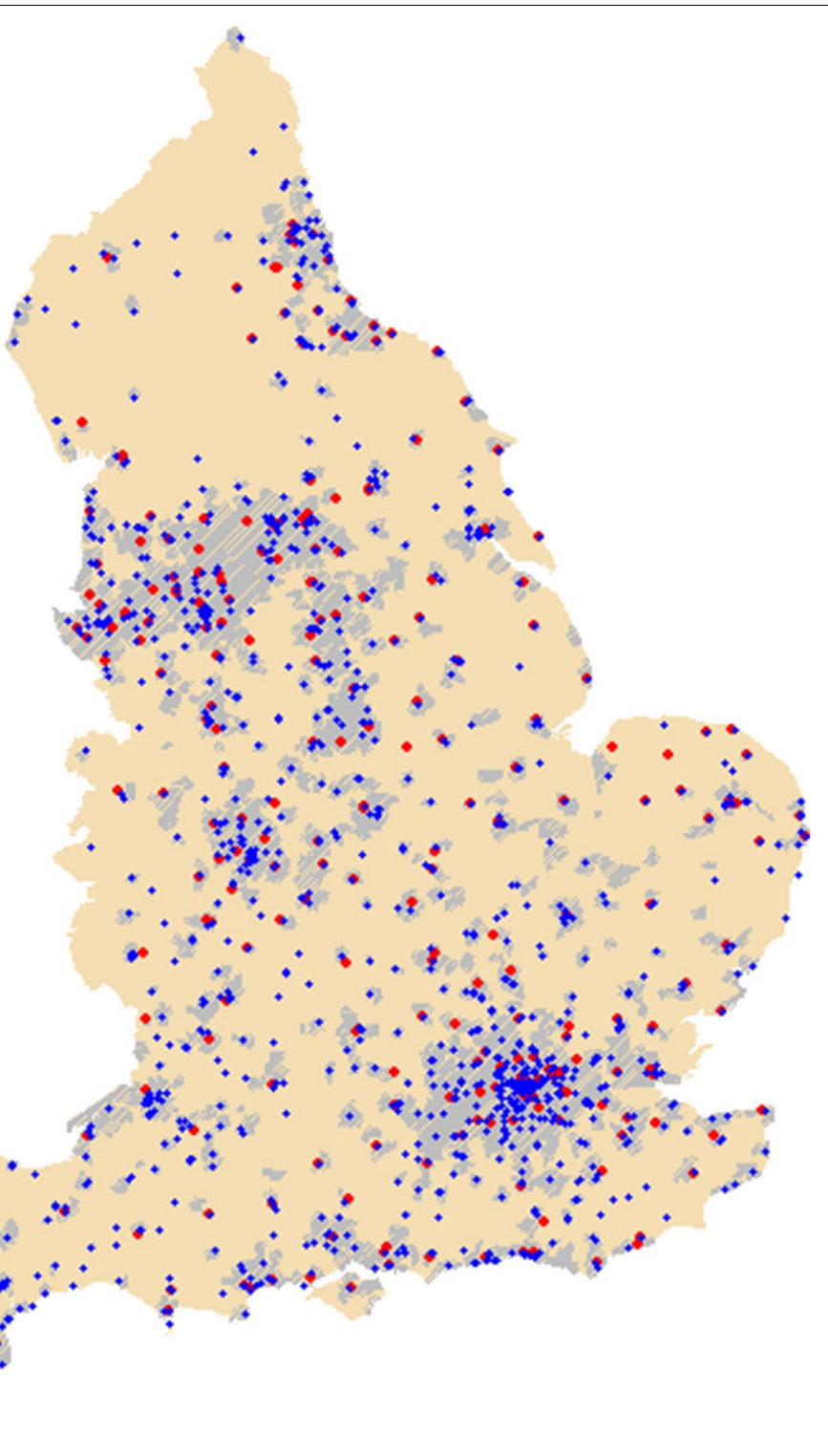

Fig. 1 Geographic distribution of inpatient PEoLC Facilities in England, UK. The digital boundary file contains National Statistics data@ Crown copyright and database right (2016) and contains Ordnance Survey data@ Crown copyright and database (2016)

layer [41]. All datasets were checked and cleaned for errors and the final datasets comprised of 184 adult inpatient hospices and 1226 hospitals.

\section{Outcome, explanatory and confounding variables}

Place of death was the outcome variable categorised into five groups; hospital, hospice, home, care home and other places. In our model, we focussed on the top three commonplace of deaths: hospices, hospital, and home [42].

Potential geographic access was the explanatory variable, categorised into four groups $(0-10 \mathrm{~min}, 10-30 \mathrm{~min}$,
$30-50 \mathrm{~min}$ and over $50 \mathrm{~min}$ ). Access was analysed as a categorical variable to facilitate interpretation and comparison of results between models. Potential geographic access was derived by measuring drive times from patients' place of usual residence to the nearest inpatient PEoLC facility. We accounted for differences in speed limits of various road types (e.g. A-roads, B-roads, Minor roads and Motorways). Calculation of drive time was completed in ArcGIS 10.5 using the Network Analyst extension [43]. The process involved estimating travel times between sets of origin locations (i.e. patients' 
residential locations) and destinations (Inpatient PEoLC facilities) along a road network. Results are stored in an origin-destination matrix, consisting of drive time values from each patient's place of residence to hospitals (1226 by 430,467$)$ and hospices $(184$ by 430,467$)$. Minimum drive time values calculated from $\mathrm{O}-\mathrm{D}$ matrices were linked to patients' records as proxy estimates of patients' access. Estimation of drive times from patients' residential address offered a detailed analysis of patients' potential geographic access to PEoLC facilities, compared to area-based access measures used in other PEoLC accessibility studies.

Confounding variables comprised of age at death (2554, 55-64, 65-74, 75-84 and 85+), gender (male and female), marital status (married, single, divorced, widowed and not given or others), CoD, NCoDs, Index for Multiple Deprivation (IMD), and region of residence. IMD was used as a proxy for patients' socioeconomic status. It is an area-based measure of deprivation, with domains in income, employment, education skills, training, health, disability, housing/services barriers and living environment. The IMD score for each patient was derived by linking patients' residential postcode to their Lower Super Output Areas (LSOAs) of residence, this was calculated for all LSOAs. LSOAs are census units with an average population of 1500 people [44]. The IMD was based on data for 2010 and was grouped into quintiles of 1 (most deprived) to 5 (least deprived). CoD and NCoDs were based on the 10th revision of the International Classification of Diseases (ICD-10) coding systems (Table 1).

\section{Statistical analysis}

Data on patients' socio-demographic and clinical characteristics were described using percentages. Maps were used to visualise geographic access in urban and rural areas. Maps were derived from the aggregates of individual-level median drive time to inpatient PEoLC facilities in each Clinical Commission Group (CCG). CCGs are planning regions for commissioning health services in England, UK [45]. There were 211 CCGs in England in 2014.

A modified Poisson regression model with robust error variances [46] was applied to evaluate the association between geographic access and place of death. Two models were developed for patients in rural and urban areas. Model 1 compared the association between geographic access to hospice, for hospice death (1) versus home death (0). Model 2 compared the association between geographic access to hospitals, for hospital death (1) versus home death (0). Only deaths falling within comparison groups were included in the models.
Models were adjusted for patients' socio-demographic and clinical characteristics (Table 1). The magnitude of the association between geographic access and place of death were described using adjusted prevalence ratios (APRs) and 95\% Confidence Intervals (CIs). Model goodness of fit was based on the difference between modelbased estimate and actual data. All models showed a statistically significant reduction in deviance values $(p<0.001)$, suggesting an adequate fit to the data. All statistical analyses were completed in R. version 3.3.1 (www.r-project.org).

\section{Results}

Patients' socio-demographic characteristics

After removing invalid drive time values, with zero drive time from hospice and hospitals (approximately 0.3\%), the final dataset consisted of 430,467 adult deaths. More than three-quarters of the deaths occurred in urban areas (79.9\%) and about one-fifth (20.1\%) in rural areas. Hospital was the most common place of death in rural (43.1\%) and urban areas (48.4\%). Proportions of death increased with increasing age in rural and urban areas, with patients aged 85 and over accounting for the largest proportion of death in rural (43.2\%) and urban areas (39.7\%). Cancer accounted for $30.1 \%$ of deaths in rural and $30.4 \%$ in urban areas. Cardiovascular Diseases (CVDs) accounted for an almost equal proportion of deaths in urban (21.1\%) and rural areas (21.0\%). Deaths from Chronic Obstructive Pulmonary Disorders (COPDs) were marginally higher in urban areas (5.9\%), compared to rural areas (5.7\%). Deaths varied remarkably across regions. The South West (18.7\%) and the South East (18.6\%) were the regions with most rural deaths. London (0.3\%) and the North East (5.8\%) had the lowest proportion of deaths in rural areas.

\section{Geographic access to inpatient PEoLC facilities in urban and rural areas}

There are distinct rural-urban differences in geographic access to inpatient PEoLC facilities across CCGs in England (Fig. 2). Overall, patients in the urban area had better geographic access, compared to rural areas (Table 1). In terms of access by inpatient PEoLC facility (Table 1), patients who died in the hospital had relatively easier access to hospital in both rural (median drive time: $9.7 \mathrm{~min}$ ) and urban areas (median drive time: $4.5 \mathrm{~min}$ ), compared to patients who died in hospices in rural (median drive time: $17.9 \mathrm{~min}$ ) and urban areas (median drive time: $9 \mathrm{~min}$ ). 
Table 1 Patients' socio-demographic and clinical characteristics of adults who died in rural and urban areas, England $2014(N=430,467)$

\begin{tabular}{|c|c|c|c|}
\hline Variable & Value & Rural & Urban \\
\hline N (\%) & & $86,432(20.1 \%)$ & $344,035(79.9 \%)$ \\
\hline \multirow[t]{2}{*}{ Geographic access (min) } & Median drive time to hospice (minimum, maximum) & $17.9(0.16,93.9)$ & $9.02(0.01,105.4)$ \\
\hline & Median drive time to hospital (minimum, maximum) & $9.71(0.02,73.9)$ & $4.5(0.00,76.2)$ \\
\hline \multirow[t]{5}{*}{ Age } & $25-54$ & 3.7 & 5.4 \\
\hline & $55-64$ & 6.7 & 8.1 \\
\hline & $65-74$ & 16.6 & 16.7 \\
\hline & $75-84$ & 29.9 & 30 \\
\hline & $85+$ & 43.2 & 39.7 \\
\hline \multirow[t]{2}{*}{ Gender } & Female & 51.3 & 52.3 \\
\hline & Male & 48.7 & 47.7 \\
\hline \multirow[t]{6}{*}{ Marital status } & Divorced & 7.8 & 10.3 \\
\hline & Married & 43.1 & 37.7 \\
\hline & Separated/dissolved & 0.1 & 0.1 \\
\hline & Single & 7.1 & 9.6 \\
\hline & Unknown/not stated & 0.3 & 0.5 \\
\hline & Widowed & 41.6 & 41.8 \\
\hline \multirow[t]{6}{*}{ Cause of death (ICD-10 codes) } & Cancers (C00-C97) & 30.1 & 30.4 \\
\hline & ${ }^{*}$ CBDs (G45-G46, 160-169) & 7.6 & 7.1 \\
\hline & ${ }^{*}$ COPDs $(J 40-J 44, J 47)$ & 5.7 & 5.9 \\
\hline & ${ }^{*}$ CVDs (100-152, 170-199) & 21 & 21.1 \\
\hline & Neurological conditions (G35-G37, G20, F02.3, G12) & 1.7 & 1.7 \\
\hline & Others (not in the above categories) & 33.8 & 33.7 \\
\hline \multirow{6}{*}{$\begin{array}{l}\text { Number of contributory } \\
\text { Causes of deaths (NCoDs) }\end{array}$} & 0 & 25.2 & 23.4 \\
\hline & 1 & 28.1 & 26.9 \\
\hline & 2 & 21.4 & 22 \\
\hline & 3 & 12.9 & 14.1 \\
\hline & 4 & 7 & 7.6 \\
\hline & $5+$ & 5.4 & 6.1 \\
\hline \multirow[t]{5}{*}{ Index of Multiple (IMD) } & 1 (most deprived) & 3.3 & 24.1 \\
\hline & 2 & 12.9 & 21.6 \\
\hline & 3 & 29.2 & 18.5 \\
\hline & 4 & 30.7 & 17.6 \\
\hline & 5 (least deprived) & 24 & 18.3 \\
\hline \multirow[t]{9}{*}{ Regions } & East & 17.6 & 9.9 \\
\hline & East-midlands & 12.5 & 8.1 \\
\hline & London & 0.3 & 12.6 \\
\hline & North-east & 5.3 & 5.8 \\
\hline & North-west & 8.3 & 16 \\
\hline & South-east & 18.6 & 16 \\
\hline & South-west & 18.7 & 9.5 \\
\hline & West midlands & 9.5 & 11.3 \\
\hline & Yorkshire and The Humber & 9.4 & 11 \\
\hline \multirow[t]{5}{*}{ Place of death } & Other place & 1.2 & 1.1 \\
\hline & Hospice & 5.5 & 6.2 \\
\hline & Home & 24.6 & 22 \\
\hline & Care home & 25.5 & 22.3 \\
\hline & Hospital & 43.1 & 48.4 \\
\hline
\end{tabular}

*Cause of death: Cardiovascular Diseases (CVDs), Chronic Obstructive Pulmonary Disease (COPD) and Cerebrovascular Disease (CBDs) 


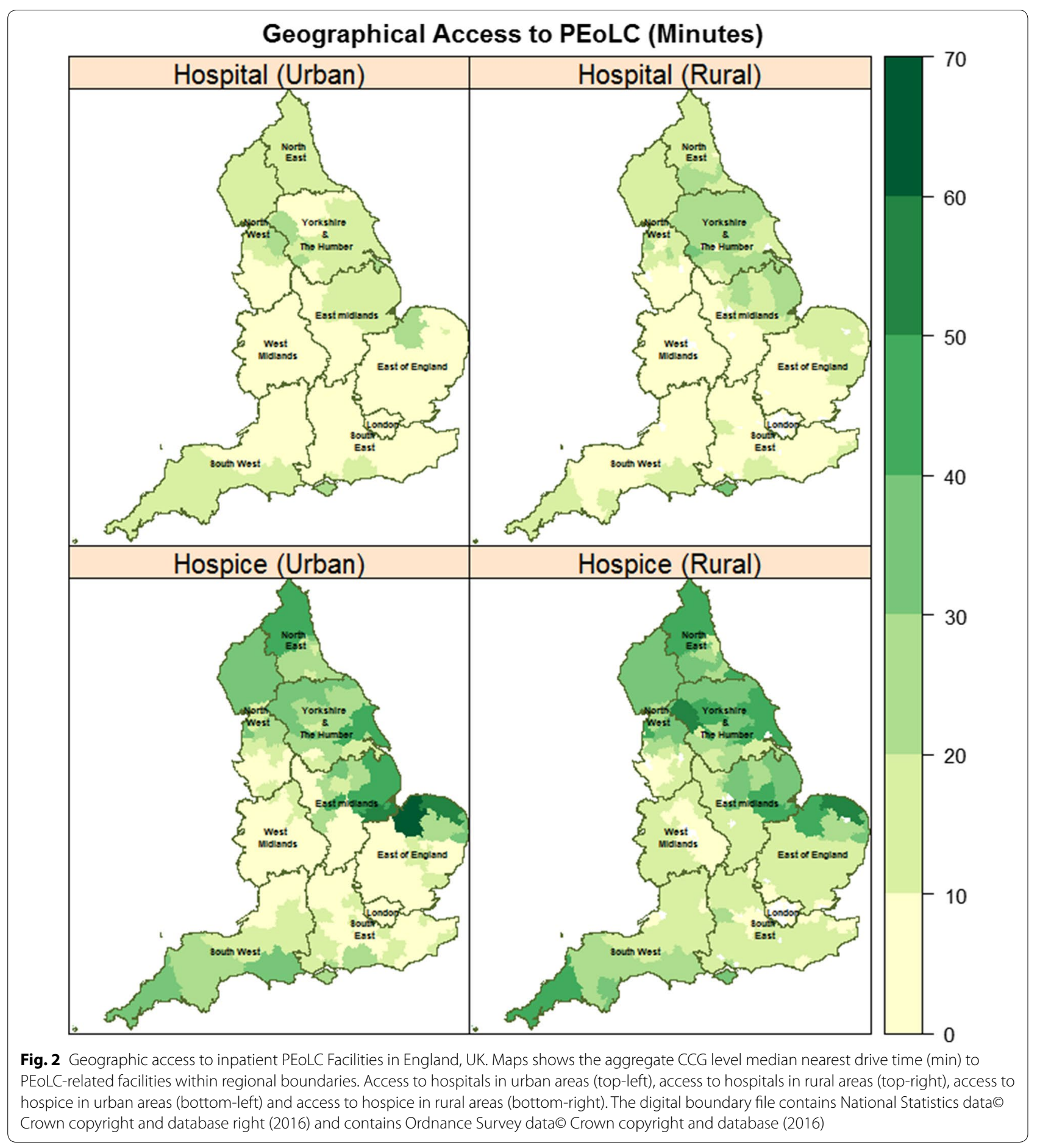

\section{Relationship between geographic access to inpatient} PEoLC facilities and Place of Death in rural and urban areas Results of multivariable analysis (Table 2), show an inverse relationship between place of death and drive time in both rural and urban areas (results of complete models are given in Additional file 1: Tables S1 and
Additional file 2: S2). In Model 1 (Hospice vs Home) there was a strong inverse association, with a doseresponse effect. In urban areas, patients who lived within a drive time range of 10-30 min from hospices were less likely to die in a hospice (APR: $0.83,95 \% \mathrm{CI}$ $0.81-0.86, p<0.001)$ compared to patients who lived 
Table 2 Association between geographic access and place of death in rural and urban areas

\begin{tabular}{|c|c|c|c|c|c|}
\hline \multirow[t]{2}{*}{ Models } & \multirow{2}{*}{$\begin{array}{l}\text { Geographic } \\
\text { access (min) }\end{array}$} & \multicolumn{2}{|l|}{ Rural } & \multicolumn{2}{|l|}{ Urban } \\
\hline & & Unadjusted PRs $(95 \% \mathrm{Cl})$ & Adjusted PR (95\% Cl) & Unadjusted PRs ( $95 \% \mathrm{Cl})$ & Adjusted PR (95\% Cl) \\
\hline 1 & \multicolumn{5}{|c|}{ Drive time to hospices } \\
\hline \multirow[t]{4}{*}{ Hospice versus home } & 0-10 min & Ref & Ref & Ref & Ref \\
\hline & $10-30 \mathrm{~min}$ & $0.77(0.72-0.81)^{* * *}$ & $0.80(0.76-0.85)^{* * *}$ & $0.84(0.82-0.86)^{* * *}$ & $0.83(0.81-0.86)^{* * *}$ \\
\hline & $30-50 \mathrm{~min}$ & $0.57(0.52-0.62)^{* * * *}$ & $0.64(0.58-0.70)^{* * * *}$ & $0.73(0.70-0.77)^{* * * *}$ & $0.74(0.70-0.77)^{* * *}$ \\
\hline & Over 50 min & $0.42(0.36-0.48)^{* * * *}$ & $0.49(0.43-0.56)^{* * * *}$ & $0.47(0.40-0.54)^{* * * *}$ & $0.50(0.43-0.59)^{* * *}$ \\
\hline 2 & \multicolumn{5}{|c|}{ Drive time to hospitals } \\
\hline \multirow[t]{4}{*}{ Hospital versus home } & $0-10 \mathrm{~min}$ & Ref & Ref & Ref & Ref \\
\hline & $10-30 \mathrm{~min}$ & $0.99(0.97-1.00)^{*}$ & $0.98(0.96-0.99)^{* * *}$ & $0.98(0.97-0.99)^{* * *}$ & 0.99 (0.99-1.00). \\
\hline & $30-50 \mathrm{~min}$ & $0.99(0.96-1.02)$ & $0.95(0.92-0.98)^{* *}$ & $0.96(0.93-0.98)^{* *}$ & $0.97(0.95-1.00)^{*}$ \\
\hline & Over 50 min & $0.80(0.58-1.12)$ & $0.79(0.59-1.06)$ & $0.94(0.82-1.09)$ & $0.98(0.86-1.13)$ \\
\hline
\end{tabular}

Prevalence ratios (PRs) were estimated from modified poisson regression. PR $>1$ indicates a higher likelihood of death at hospice or hospital compared to the reference category. PR $<1$ suggest lower likelihood of hospice or hospital death compared to the reference category (Ref - reference group). Adjusted PRs were derived by adjusting for age, cause of death (COD), gender, marital status, Index of Multiple Deprivation (IMD), number of contributory cause of deaths (NCODs) and regions. Triple asterisks $\left({ }^{* *}\right)$ denotes $p$ value less than 0.001 , double asterisks $\left(^{* *}\right)$ is $p$ value less than 0.01 and Single asterisk $(*)$ is less than 0.05 and a dot $($.$) means p$ value less than 0.1

closer (0-10 $\mathrm{min})$. Successive increases in drive time from $10 \mathrm{~min}$ to $30-50 \mathrm{~min}$ (APR. 0.74, 95\% CI 0.70-0.77, $p<0.001$ ) or over $50 \mathrm{~min}$ (APR. $0.50,95 \%$ CI $0.43-0.59$, $p<0.001)$, resulted in corresponding reductions in likelihoods of hospice death. The effects were greater in rural areas for all drive time categories (APR: 0.80, 95\% CI $0.76-0.85, p<0.001[10-30 \mathrm{~min}]$; APR. $0.64,95 \%$. CI $0.58-0.70, p<0.001$ [30-50 min] and APR. 0.49, 95\% C I $0.43-0.56, p<0.001$ [Over $50 \mathrm{~min}$ ]).

The effect of drive time on hospital deaths (Model 2: Hospital vs Home), was similar to those obtained for hospice in rural and urban areas. In urban areas, patients who lived more than $10 \mathrm{~min}$ drive time from a hospital location (10-30 min APRs. 0.99, 95\% CI 0.99-1.00, $p=0.057$; $30-50 \mathrm{~min}$, APRs. 0.97, 95\% CI 0.95-1.00, $p=0.032$; over $50 \mathrm{~min}$, APR. $0.9895 \%$ CI $0.86-1.13$, $p=0.79)$, were less likely to die in a hospital, compared to patients who lived closer (i.e. $0-10 \mathrm{~min}$ ). The magnitudes of the APRs (APRs. 0.98, 0.96-0.99, $p<0.001$ [10-30 min]; APRs. 0.95; 95\% CI 0.92-0.98, $p<0.01$ [30$50 \mathrm{~min}$ ]; APRs. $0.79,0.59-1.06, p=0.11$ [Over $50 \mathrm{~min}$ ]) were lesser in rural areas.

\section{Discussion}

The results of this population-based study show that geographic access to inpatient PEoLC-facilities is associated with place of death, with the magnitude of the effect being greater in rural areas compared to urban areas. These findings warrant the formulation of end-of-life care policies that account for differences in settlement types, such that outreach home care may become especially important in more rural areas.
Patients in rural and urban areas who lived more than 10 min away from hospices were less likely to die in a hospice, compared to those who lived within $10 \mathrm{~min}$, drive time. Even after adjusting for patients' sociodemographic and clinical characteristics (Model 1), the associations remained statistically significant $(p<0.001)$. Similar results were found in the association between geographic access to hospital and place of death. However, the gradient was not as steep as those obtained in geographic access to hospice. Our results are consistent with the findings of Gatrell and colleagues [35]. Their study showed that the likelihood of dying in a hospice or hospital was inversely associated with distance, using cancer only data in the North West England and, controlled for a limited number of confounders. By contrast, our study used large national death data (consisting of deaths from Cancers and Non-cancers) and adjusted for patients' socio-demographic and clinical characteristics including, $\mathrm{CoD}, \mathrm{NCoDs}$ and regions of residence. Although, the inclusion of covariates did not change the direction of the association, but the strength of the association was reduced slightly in the adjusted model (Table 2). This implies that ignoring these important covariates may exaggerate the effect of geographic access to PEoLC in place of death.

Our results show a clear dose-response relationship between geographic access to hospice and hospice deaths in urban and rural areas (Model 1). This suggests that greater drive time from a hospice leads to a corresponding decline in the likelihood of dying in that hospice. This attenuating effect of drive time on the likelihood of hospice death, suggests that the further away patients lived from a hospice, the lower the chance of hospice 
death. The same is true for geographic access to hospitals (Model 2). However, we would exercise caution against the over interpretation that this means that patients who lived in rural areas and died at home had less good care, as it is possible that areas with limited geographic access to inpatient facilities are served by community-based care, especially in rural areas.

The differences in the magnitude of the effect between urban and rural areas underscores the importance of place of settlement on where people die. One possible reason for variations may be due to differences in the spatial organisation of inpatient PEoLC facilities in rural and urban areas. As Fig. 1 shows, hospitals and hospices are clustered in urban areas, compared to rural areas. This means that in rural areas, patients experience longer drive time to hospices and hospitals. In addition, the map of aggregate CCG level, median drive time to inpatient PEoLC facilities (Fig. 2) shows that patients in rural areas travel longer distances to use hospice and hospital services, compared to their urban counterparts. Northern Norfolk around King's Lynn was a notable exception (Fig. 2, bottom-left). Drive time to hospice in the area appears to be greater in urban areas (approximately above $60 \mathrm{~min}$ from hospice locations) compared to rural areas. This is perhaps due to limited number of hospices in relation to the proportion of urban deaths (Fig. 1). Further investigation is needed to understand the cause of the drive time anomaly in Northern Norfolk.

Rurality is associated with an increased chance of home death. It will be important to determine whether this is because of choice or because of access problems to inpatient facilities [47], which can be misinterpreted as choice. Several studies have reported similar rural-urban disparities in health services provision/access [7, 28, 48]. Rural-urban disparities in geographic access have implications for services utilisation $[7,8,49,50]$ and can lead to late diseases diagnosis amongst rural dwellers [51, 52].

The findings of this study have important implications for service improvement, planning and delivery, particularly in terms of the way community-based palliative care services are configured to meet needs. Community hospice teams should expand their service catchments farther from hospice and hospital locations, especially in rural areas where drive time to facilities is greater. There is evidence that such a targeted service delivery approach can foster service uptake in health service deficit areas [53]. It could be argued that community services may also need to be increased in urban areas as the higher rate of death in hospitals may not be reflective of choice, but rather a decreased chance of dying at home due to insufficient community services.

Although our study used data from England, UK findings are applicable to other countries. The findings can assist health policy makers in understanding the role of geographic accessibility in place of death and for planning service improvement interventions especially in underserved areas (e.g. rural areas). Findings can feed into local end of life policies and strategies of other countries to improve equity in health care delivery for people nearing end-of-life. For example, formulation of policies to foster decentralisation of inpatient PEoLC services from urban areas can alleviate the challenge of geographic access in rural areas. One way this can be achieved is by introducing non-institutionalised services-e.g. hospice-at-home services. Technology-enhanced care such as telehealth services [54] or video conferencing can improve access to specialist palliative care in remote or rural areas. For example, video conferencing has been piloted in Orkney Scotland Hospice [47] and it is currently being used to address the problem of access in remote communities in Scotland [55]. Increasing services capacity, such as the provision of inpatient hospice beds, and the introduction of initiatives that foster collaborative palliative service delivery across care settings-e.g. delivery of hospice services at care homes or hospitals, should be adopted. There is evidence that such collaborative involvement or partnership can increase out-of-hospital death [56] and improve access to specialist palliative care services [57].

\section{Strength and limitations of study}

To our knowledge, this is the first study to comprehensively examine rural-urban differences in the association between geographic access to inpatient PEoLC facilities and place of death using large individual-level death data. The main strength of this study is the use of individual-level data as the unit of analysis of geographic access. Deriving access from individual-level data is less prone to aggregation errors, compared to aggregate level access measures used in other PEoLC accessibility studies [23, 24, 28, 31, 32], that assumes that individuals within in a geographical area have the same accessibility scores [58]. The ONS death registry provides spatially disaggregate data with patientlevel information, such as age, gender, the main cause and contributory causes of death. Linkage of the data with an area-level settlement and socio-economic data enabled the exploration of urban and rural differences and the role of access in place of death. Similar databases can be used in other developed and developing countries. While the quality and completeness of death registers is a fundamental issue in developing countries, a national representative sample of existing death data linked with local census estimates and health facility location data could be used to analyse rural and urban difference in access. The study made important contribution by exploring how access varies 
between urban and rural settlements, while adjusting for a wide range of covariates that may confound the association between geographic access and place of death not considered in previous study on geographic access and place of death. This is important for more robust service improvement interventions and policy formulation.

Geographic access was derived by measuring the drive time from patients' place of residence to the nearest inpatient PEoLC facility. Drive time was used because it is a more appropriate measure of travel efforts $[59,60]$. It provides a better proxy estimate of geographic access compared to straight-line or 'crowfly distance', although the latter has been shown to be highly correlated with other measures of access [33, 61, 62]. Nevertheless, results should be interpreted with caution, as drive time may give a false impression that patients have access to only one mode of transportation (private car or public transport). In addition, the calculation of drive time was based on fixed speed limits without consideration of road conditions. Studies have shown that speed limits are variable depending on the time of day [63] and seasons [64]. Prospective studies should consider these factors when measuring drive time. Another strength of this study relates to the use of prevalence ratios to estimate relative risk, instead of odd ratios. The latter has been shown to exaggerate effect size when the outcome of interest is common [46].

Our study has some limitations. Due to data processing constraints, we calculated drive time by splitting the datasets based on patients' region of residence. This involved calculating drive time for each region separately and recombining the datasets. Using this stratified approach leads to edge-effect error [65] as cross-regional movement is not taken into account. For example, patients living in the border of regions may access services outside their region of residence which are closest to them.

Furthermore, geographic access was quantified based on the assumption that patients will visit facilities nearest to them. However, in practice, patients' usage of service encompasses more than geographic proximity [66]. Access is a multifaceted concept consisting of both geographical and non-geographical factors [17] (e.g. availability, affordability, accommodation, acceptability). Analysing access in the context of geographic dimension ignores non-geographical factors (e.g. the number of beds, workforce and, patients' preference) that can influence access to inpatient PEoLC facilities. For example, it was not possible to quantify community based generic or specialist palliative care facilities to understand whether they are greater in rural areas. Future studies should incorporate these non-geographical aspects in the model to gain a holistic picture of the role of geographic access in place of death.

\section{Conclusion}

Findings from this study have shown that geographic access is an important determinant of place of death and that the size of the effect differs in rural and urban areas. These results highlight the need for the formulation of end-of-life policies/strategies that consider differences in settlements types. Overall, findings from this study showed that rural dwellers are less likely to die at PEoLC inpatient facilities, compared to their urban counterparts. Findings can feed into local end-of-life policies and strategies of other countries to improve equity in health care delivery for people nearing end of life. The utility of individual-level death data as a unit of analysing geographic access permitted a detailed examination of urban/rural differences in the association between geographic access to inpatient PEoLC and place of death.

\section{Additional files}

Additional file 1. Table S1.xls: (Additional file Table 1) Association between Drive time to hospices and Place of death in Rural and Urban areas in England (2014), adjusting for clinical and socio-demographic characteristics of patients.

Additional file 2. Table S2.xls: (Additional file Table 2) Association between Drive time to hospitals and Place of death in Rural and Urban areas in England (2014), adjusting for clinical and socio-demographic characteristics of patients.

\section{Abbreviations}

PEOLC: Palliative and end-of-life care facilities; IMD: Index for Multiple Deprivation; CoD: Underlying Cause of Death; NCoD: Number of contributory causes of death; LSOAs: Lower Super Output Areas; ICD-10: International Classification of Diseases 10th revision; CCGs: Clinical Commission Groups (CCGs); APRs: Adjusted Prevalence Ratios; Cl: Confidence Interval; CVDs: Cardiovascular Diseases; COPD: Chronic obstructive pulmonary disease; CBDs: Cerebrovascular diseases; ONS: Office for National Statistics; RUC: Rural-Urban classification; O-D: Origin-Destination matrix.

\section{Authors' contributions}

WG, IJH, JV, PG. obtained funding. EC and WG conceived the idea for the study and designed the analysis plan; EC and WG implemented the analysis plan. WG and EC were responsible for liaison with the Office for National Statistics (ONS), data checking, recoding and merging of datasets. EC wrote the first draft of the manuscript, with significant input from WG. All authors critically reviewed the manuscript and approved the final version for submission. All authors read and approved the final manuscript.

\section{Author details}

${ }^{1}$ Department of Palliative Care, Policy and Rehabilitation, Cicely Saunders Institute, King's College London, Bessemer Road, Denmark Hill, London SE5 9PJ, UK. ${ }^{2}$ Knowledge and Intelligence (South West), National End of Life Care Intelligence Network, Public Health England, Grosvenor House, 2 Rivergate, Temple Quay, Bristol BS1 6EH, UK. ${ }^{3}$ Monitoring Analytics (Mental Health, Learning Disability and Substance Misuse), Care Quality Commission (CQC), 151 Buckingham Palace Road, London SWIW 9SZ, UK. ${ }^{4}$ Royal Marsden NHS Hospital Trust, London SW3 6JJ, UK. ${ }^{5}$ Hospice UK, 34-44 Britannia Street, London WC1X 9JG, UK. 


\section{Acknowledgements}

The Guide_Care Services project is a large scale observational study investigating the role of service factors in place of death funded by the National Institute for Health Research, Health Services and Delivery Research Programme (NIHR HS\&DR, 14/19/22), led by Cicely Saunders Institute, Department of Palliative Care, Policy \& Rehabilitation at King's College London, UK. It is a follow-on project to the Guide_Care project (NIHR HS\&DR, 14/19/22) (NIHR HS\&DR, 14/19/22). We thank all collaborators and advisors including serviceusers. Investigators: Wei Gao (co-PI), Irene J Higginson (co-PI), Julia Verne, Emma Gordon and Giovanna Polato. Project Advisory Group members: Tony Bonser, Nicola Bowtell, Kate Heaps, Jamie Jenkins, Katie Lindsey, Catherine Millington-Sanders, Rajive Mitra, Myfanwy Morgan, Carolyn Morris, Robert Mulliss, Andy Pring, Sarah Russell, Jane Smith, Claire Henry, Claudia Wells, Paula Young. Researchers: Emeka Chukwusa (Project Manager), Peihan Yu, Rebecca Wilson, Clare Pearson, Sumaya Huque. Administrative support: Halle Johnson, Daniel Gulliford, Sophie Watson, Zaynah Sheikh. We thank the data providers for this work: Office for National Statistics (ONS), the Health and Social Care Information Centre (HSCIC), Care Quality Commission (CQC), the National Council for Palliative Care (NCPC), the London Cancer Alliance (LCA), Ordnance Survey data_-C Crown Copyright and Database Right 2016. Ordnance Survey (Digimap License).

\section{Competing interests}

We declare no conflicts of interest or competing interests.

\section{Availability of data and materials}

The data that support the findings of this study are available from the Office for National Statistics (ONS), but restrictions apply to the availability of these data, which were used under the data agreement (MRP 1935/2011) for the current study, and so are not publicly available. Data are however available with special approvals from the ONS.

\section{Consent for publication}

Not applicable.

\section{Ethics and consent to participate}

Ethical approval was granted by the King's College London Research Ethics committee for secondary analysis of death registration data (Reference Number: BDM/14/15-5). Researchers (EC, GW) accessing the ONS data underwent ONS approved researcher training and were approved by the ONS. This is a study based on fully anonymised datasets and no direct patient contact, hence, no consent to participate was required.

\section{Funding}

The National Institute for Health Research, Health Services and Delivery Research Programme Guide_Care Services project (NIHR HS \& DR, 14/19/22). This project is partly supported by the National Institute for Health Research (NIHR) Collaboration for Leadership in Applied Health Research and Care (CLAHRC) South London at King's College Hospital NHS Foundation Trust, and Cicely Saunders International. The views expressed are those of the author(s) and not necessarily those of the NHS, the NIHR or the Department of Health.

\section{Publisher's Note}

Springer Nature remains neutral with regard to jurisdictional claims in published maps and institutional affiliations.

Received: 23 November 2018 Accepted: 27 April 2019

Published online: 06 May 2019

\section{References}

1. Gao W, et al. A population-based conceptual framework for evaluating the role of healthcare services in place of death. Healthcare (Basel). 2018;6(3):107. https://doi.org/10.3390/healthcare6030107.

2. Higginson $\mathrm{IJ}$, et al. Which patients with advanced respiratory disease die in hospital? A 14-year population-based study of trends and associated factors. BMC Med. 2017;15, 19. https://doi.org/10.1186/s1291 6-016-0776-2
3. Cohen J, et al. Access to palliative care services in hospital: a matter of being in the right hospital Hospital charts study in a Canadian city. Palliat Med. 2012;26(1):89-94. https://doi.org/10.1177/0269216311408992.

4. Ziegler LE, et al. Is palliative care support associated with better quality end-of-life care indicators for patients with advanced cancer? A retrospective cohort study. BMJ Open. 2018;8(1), e018284. https://doi. org/10.1136/bmjopen-2017-018284.

5. WHO, Strengthening of palliative care as a component of comprehensive care throughout the life course, WH Organisation (ed) 2014.

6. Goodridge D, et al. Rural/urban differences in health care utilization and place of death for persons with respiratory illness in the last year of life. Rural Remote Health. 2010;10(2):1349.

7. Virnig BA, et al. Geographic variation in hospice use prior to death. J Am Geriatr Soc. 2000;48(9):1117-25. https://doi. org/10.1111/j.1532-5415.2000.tb04789.x.

8. Virnig BA, et al. Do rural elders have limited access to medicare hospice services? J Am Geriatr Soc. 2004;52(5):731-5. https://doi.org/10.111 1/j.1532-5415.2004.52213.x.

9. Houttekier D, et al. Place of death in metropolitan regions: metropolitan versus non-metropolitan variation in place of death in Belgium, The Netherlands and England. Health Place. 2010;16(1):132-9. https://doi. org/10.1016/j.healthplace.2009.09.005.

10. Higginson IJ, et al. Which patients with advanced respiratory disease die in hospital? A 14-year population-based study of trends and associated factors. BMC Med. 2017;15(1):19. https://doi.org/10.1186/s1291 6-016-0776-2.

11. Cohen J, et al. Dying at home or in an institution: using death certificates to explore the factors associated with place of death. Health Policy. 2006;78(2):319-29. https://doi.org/10.1016/j.healthpol.2005.11.003.

12. Gomes B, Higginson IJ. Factors influencing death at home in terminally ill patients with cancer: systematic review. BMJ. 2006;332(7540):515-21. https://doi.org/10.1136/bmj.38740.614954.55.

13. Rainsford S, MacLeod RD, Glasgow NJ. Place of death in rural palliative care: a systematic review. Palliat Med. 2016;30(8):745-63. https://doi. org/10.1177/0269216316628779.

14. Gulliford M, et al. What does 'access to health care' mean? J Health Serv Res Policy. 2002;7(3):186-8. https://doi.org/10.1258/135581902760082 517.

15. Schuurman $N$, et al. Defining rational hospital catchments for non-urban areas based on travel-time. Int J Health Geogr. 2006;5(1):43.

16. Levesque J-F, Harris MF, Russell G. Patient-centred access to health care: conceptualising access at the interface of health systems and populations. Int J Equity Health. 2013;12(1):18. https://doi. org/10.1186/1475-9276-12-18.

17. Penchansky R, Thomas JW. The concept of access: definition and relationship to consumer satisfaction. Med Care. 1981;19(2):127-40.

18. Luo W, Wang F. Measures of spatial accessibility to health care in a GIS environment: synthesis and a case study in the Chicago region. Environ Plan. 2003;30(6):865-84. https://doi.org/10.1068/b29120.

19. Campbell NC, et al. Rural and urban differences in stage at diagnosis of colorectal and lung cancers. Br J Cancer. 2001;84(7):910-4. https://doi. org/10.1054/bjoc.2000.1708.

20. Murage $P$, et al. Geographical disparities in access to cancer management and treatment services in England. Health Place. 2016;42:11-8. https:// doi.org/10.1016/j.healthplace.2016.08.014.

21. Jones AP, et al. Geographical access to healthcare in Northern England and post-mortem diagnosis of cancer. J Public Health. 2010;32(4):532. https://doi.org/10.1093/pubmed/fdq017.

22. Dejardin O, et al. The influence of geographical access to health care and material deprivation on colorectal cancer survival: evidence from France and England. Health Place. 2014;30:36-44. https://doi.org/10.1016/j.healt hplace.2014.08.002.

23. Gatrell AC, Wood DJ. Variation in geographic access to specialist inpatient hospices in England and Wales. Health and Place. 2012;18(4):832-40. https://doi.org/10.1016/j.healthplace.2012.03.009.

24. Wood DJ, Clark D, Gatrell AC. Equity of access to adult hospice inpatient care within north-west England. Palliat Med. 2004;18(6):543-9. https:// doi.org/10.1191/0269216304pm892oa.

25. Currow DC, et al. Referral patterns and proximity to palliative care inpatient services by level of socio-economic disadvantage. A national study 
using spatial analysis. BMC Health Serv Res. 2012;12(1):424. https://doi. org/10.1186/1472-6963-12-424.

26. Virnig BA, et al. Access to home-based hospice care for rural populations: identification of areas lacking service. J Palliat Med. 2006;9:1292-9.

27. Lindley LC, Edwards SL. Geographic access to hospice care for children with cancer in Tennessee, 2009 to 2011. Am J Hosp Palliat Med ${ }^{\circledR}$. 2014;32(8):849-54. https://doi.org/10.1177/1049909114543641.

28. Carlson MDA, et al. Geographic access to hospice in the United States. J Palliat Med. 2010;13(11):1331-8. https://doi.org/10.1089/jpm.2010.0209.

29. Crooks VA, et al. Refining a location analysis model using a mixed methods approach: community readiness as a key factor in siting rural palliative care services. J Mixed Methods Res. 2010;5(1):77-95. https://doi. org/10.1177/1558689810385693.

30. Cinnamon J, Schuurman N, Crooks VA. Assessing the suitability of host communities for secondary palliative care hubs: a location analysis model. Health Place. 2009;15(3):822-30. https://doi.org/10.1016/j.healt hplace.2009.01.003.

31. Cinnamon J, Schuurman N, Crooks VA. A method to determine spatial access to specialized palliative care services using GIS. BMC Health Serv Res. 2008;8(1):140. https://doi.org/10.1186/1472-6963-8-140.

32. Schuurman $\mathrm{N}$, et al. A comparative analysis of potential spatio-temporal access to palliative care services in two Canadian provinces. BMC Health Serv Res. 2015;15(1):270. https://doi.org/10.1186/s12913-015-0909-x.

33. Apparicio $\mathrm{P}$, et al. Comparing alternative approaches to measuring the geographical accessibility of urban health services: distance types and aggregation-error issues. Int J Health Geogr. 2008;7(1):7. https://doi. org/10.1186/1476-072X-7-7.

34. Hewko J, Smoyer-Tomic KE, Hodgson MJ. Measuring neighbourhood spatial accessibility to urban amenities: does aggregation error matter? Environ Plan A Econ Space. 2002;34(7):1185-206. https://doi.org/10.1068/ a34171.

35. Gatrell AC, et al. Place of death: analysis of cancer deaths in part of North West England. J Public Health Med. 2003;25(1):53-8.

36. ONS, Office for national statistics - postcode directory. 2016 [cited 22 November 2016; Available from: http://geoportal.statistics.gov.uk/.

37. Bibby P, Brindley P, The 2011 rural-urban classification for small area geographies: a user guide and frequently asked questions (v1.0). Government statistical Service, 2013.

38. Digital N, Hospitals. 2015 [cited 22 November 2016; Available from: https ://data.gov.uk/dataset/f4420d1 c-043a-42bc-afbc-4c0f7d3f1620/hospitals.

39. NEoLCIN, National end of life care intelligence network-end of life care profiles. N.D [cited 22 November 2016; Available from: www.endoflifec are-intelligence.org.uk/view?rid $=470$.

40. UK Ha, Directory of UK Hospices, by county. 2015 [cited 22 November 2016; Available from: http://www.hospiceaid.org.uk/images/guide_to_ hospice_aid/hospicedirectorybycounty.pdf.

41. MasterMap OS, OS MasterMap ${ }^{\circledR}$ integrated transport network layer [GML2 geospatial data], EDOS Service (ed) 2016.

42. Gao W, et al. Changing patterns in place of cancer death in England: a population-based study. PLOS Med. 2013;10(3):1-10. https://doi. org/10.1371/journal.pmed.1001410.

43. Esri, ArcGIS desktop: release 10 May 2016.

44. Martin D. Understanding the social geography of census undercount. Environ Plan A. 2010;42(11):2753-70. https://doi.org/10.1068/a43123.

45. Naylor $\mathrm{C}$, et al. Clinical commissioning groups: Supporting improvement in general practice?. London: The King's Fund; 2013. p. 69.

46. Zou G. A modified poisson regression approach to prospective studies with binary data. Am J Epidemiol. 2004;159(7):702-6.
47. Scotland A, Review of palliative care services in Scotland. 2008.

48. Lin Y-H, et al. Trend of urban-rural disparities in hospice utilization in Taiwan. PLoS ONE. 2013;8(4):1-6. https://doi.org/10.1371/journ al.pone.0062492.

49. Naruse T, et al. Measurement of special access to home visit nursing services among Japanese disabled elderly people: using GIS and claim data. BMC Health Serv Res. 2017;17(1):377. https://doi.org/10.1186/s1291 3-017-2322-0.

50. Himes $C L$, Rutrough TS. Differences in the use of health services by metropolitan and nonmetropolitan elderly. J Rural Health. 1994;10(2):80-8.

51. Liff JM, Chow WH, Greenberg RS. Rural-urban differences in stage at diagnosis. Possible relationship to cancer screening. Cancer. 1991;67(5):1454-9.

52. Trepka MJ, et al. Late HIV diagnosis: differences by rural/urban residence, Florida, 2007-2011. AIDS Patient Care STDS. 2014;28(4):188-97. https:// doi.org/10.1089/apc.2013.0362

53. Betts G, Betts J. Establishing a child health clinic in a deprived area. Health Visit. 1990;63(4):122-4.

54. McCall K, et al. Perceptions of the use of a remote monitoring system in patients receiving palliative care at home. Int J Palliat Nurs. 2008;14(9):426-31. https://doi.org/10.12968/ijpn.2008.14.9.31121.

55. Improvement $\mathrm{ClaH}$, Services for older people in Orkney. 2017; p. 1-68.

56. Houttekier $D$, et al. Involvement of palliative care services strongly predicts place of death in Belgium. J Palliat Med. 2010;13(12):1461-8. https:// doi.org/10.1089/jpm.2010.0279.

57. Saxby C, et al. P-149 Nurse led hospice beds-improving access to specialist hospice nursing care for all patients at the end of life. BMJ Support Palliat Care. 2015;5:A53-4

58. Kwan M-P. Space-time and integral measures of individual accessibility: a comparative analysis using a point-based framework. Geogr Anal. 1998:30(3):191-216. https://doi.org/10.1111/j.1538-4632.1998.tb00396.x.

59. Haynes $R$, et al. Validation of travel times to hospital estimated by GIS. Int J Health Geogr. 2006:5:40. https://doi.org/10.1186/1476-072X-5-40.

60. Martin D, et al. Geographical aspects of the uptake of renal replacement therapy in England. Int J Popul Geogr. 1998;4(3):227-42. https ://doi.org/10.1002/(SICI)1099-1220(199809)4:3\%3c227:AID-IJPG8 5\%3e3.0.CO;2-\%23.

61. Pearson C, et al. Measuring geographical accessibility to palliative and end of life (PEOLC) related facilities: a comparative study in an area with well-developed specialist palliative care (SPC) provision. BMC Palliat Care. 2017;16(1):14. https://doi.org/10.1186/s12904-017-0185-0.

62. Shahid $\mathrm{R}$, et al. Comparison of distance measures in spatial analytical modeling for health service planning. BMC Health Serv Res. 2009;9(1):200 https://doi.org/10.1186/1472-6963-9-200.

63. Bernhard F, Martin G, Stefan G. Time-varying travel times in vehicle routing. Transp Sci. 2004;38(2):160-73. https://doi.org/10.1287/trsc.1030.0062.

64. Makanga PT, et al. Seasonal variation in geographical access to maternal health services in regions of southern Mozambique. Int J Health Geogr. 2017;16(1):1. https://doi.org/10.1186/s12942-016-0074-4

65. Gao F, et al. Does the edge effect impact on the measure of spatial accessibility to healthcare providers? Int J Health Geogr. 2017;16(1):46. https:// doi.org/10.1186/s12942-017-0119-3.

66. Comber AJ, Brunsdon C, Radburn R. A spatial analysis of variations in health access: linking geography, socio-economic status and access perceptions. Int J Health Geogr. 2011;10(1):44. https://doi. org/10.1186/1476-072X-10-44. 